\title{
AVALIAÇÃO DO IMPACTO DO TEOR DE ENXOFRE DE CARVÕES METALÚRGICOS NOS SEUS VALORES EM USO*
}

Michel Rodrigues Leite ${ }^{1}$ Luiz Fernando Andrade de Castro ${ }^{2}$

\section{Resumo}

A origem do enxofre no aço está relacionada com a utilização do coque para a produção de ferro-gusa. Para a maioria dos aços são requeridos baixos teores de enxofre porque este elemento afeta a isotropia das propriedades mecânicas, notadamente a tenacidade. Este trabalho tem como objetivo avaliar o impacto do teor de enxofre dos carvões metalúrgicos no seu valor em uso. Valor em uso é o preço máximo que uma determinada empresa está disposta a pagar por um material de substituição, sem deteriorar os resultados econômicos. A dessulfuração de ferrogusa e o impacto do teor de enxofre dos carvões no seu valor em uso foram avaliados considerando utilização de escórias sintéticas. Os resultados mostraram que para 4 das 5 condições avaliadas os valores em uso dos carvões foram maiores que a referência do mercado. Considerando o preço do carvão de referência igual a $120,00 \$ / t$ o valor em uso referência do mercado, assumindo $0,10 \%$ de enxofre adicional no carvão é de 118,80 \$/t. Nas simulações de dessulfuração com escória sintética os valores em uso foram estimados entre 118,55 \$/t e 119,34 \$/t.

Palavras-chave: Dessulfuração; Enxofre; Valor em uso; Escória sintética.

\section{IMPACT ASSESSMENT OF SULFUR CONTENT OF METALLURGICAL COALS ON THEIR VALUES IN USE}

\begin{abstract}
The origin of sulfur in steel is mainly related to the use of coke on pig iron production in blast furnaces. For most steels low sulfur levels are required because this element affects the isotropy of mechanical properties, especially toughness. This work aims to assess the impact of the sulfur content of metallurgical coals on their value in use. Value in use is the maximum price that a particular company is willing to pay for a replacement material without deteriorating economic results. The pig iron desulfurization and the impact of sulfur content of coals on its value in use were evaluated considering use of synthetic slags. The results showed that in the 4 of the 5 evaluated conditions values in use of coals were higher than the market reference. Considering the price of the reference coal equal to $120,00 \$ / t$ the market reference of value in use assuming additional sulfur of $0,10 \%$ in the coal is $118,80 \$ / \mathrm{t}$. In the simulations of desulfurization with synthetic slag values in use are estimated between $118,55 \$ / t$ and $119,34 \$ / t$.
\end{abstract}

Keywords: Desulfurization; Sulfur; Value in use; Synthetic slag. 


\section{INTRODUÇÃO}

O enxofre é um elemento que está contido nas matérias-primas da fabricação do aço, especialmente o coque, e é desejado quando se procura fabricar aços de corte fácil para fabricação em série de peças. Nestes casos, devido à formação de sulfeto em camadas, melhora-se a usinabilidade do aço, possibilitando um aumento de produção do equipamento de corte. Entretanto de acordo com Susaki [1] na grande maioria dos aços, a obtenção de baixo teor de enxofre no aço é desejável porque este elemento prejudica a isotropia das propriedades mecânicas, notadamente a tenacidade. Segundo Rainer et alii [2], as especificações para aço são: (i) Aço baixo carbono comum, como vergalhões, perfis e fio-máquina, a especificação de enxofre do aço indica teores menores que 0,0250\%; (ii) Aços especiais, como LC (baixo carbono) e ULC (ultra baixo carbono), aço elétrico, chapa de estanho e a maior parte dos produtos longos as especificações estão na faixa de $0,0050 \%$ a $0,0100 \%$; (iii) Para aços ULS (ultra baixo enxofre) são exigidos teores de enxofre de $0,0010 \%$. Os aços ULS podem ser resistentes à trinca induzida por hidrogênio (HIC) e aços de alta resistência para chapas blindadas ou chapas de tanques de GNL (gás natural liquefeito).

De acordo com Pielet et alii [3] valor em uso é o preço máximo que uma determinada empresa está disposta a pagar por um material de substituição, sem deteriorar os resultados econômicos. Esta avaliação é realizada substituindo uma matéria-prima de referência pelo produto sobre o qual se deseja realizar a avaliação. $O$ valor em uso é também denominado preço de indiferença.

Segundo Rainer et alii [2], em geral os custos aumentam com o teor inicial crescente de enxofre no gusa líquido, em função do consumo de agentes de dessulfuração e custos com manutenção, lanças, nitrogênio, perdas de temperatura, perdas de ferro para a escória e custos de processamento da mesma. A figura 1 evidencia os custos totais de dessulfuração do gusa líquido.

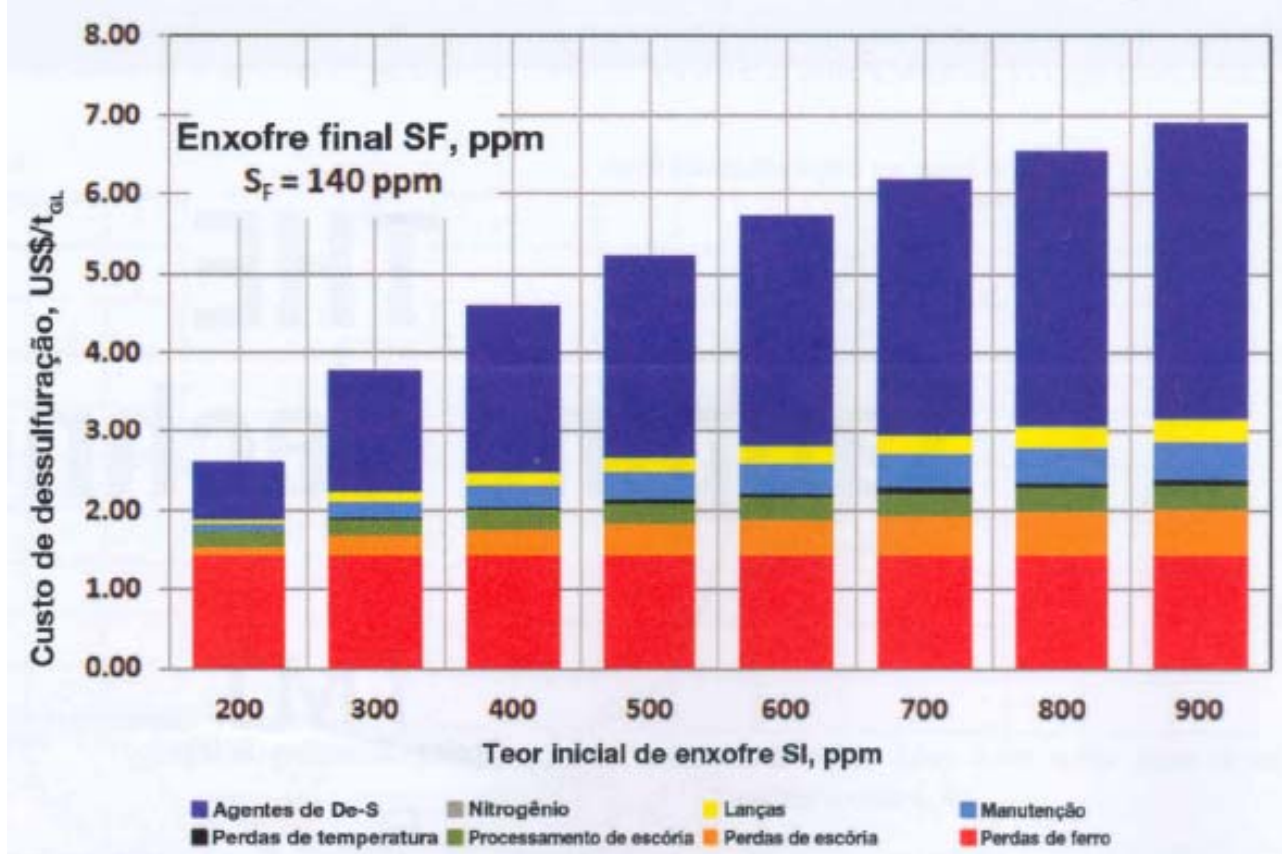

Figura 1. Estrutura dos custos de operação de dessulfuração do gusa líquido, segundo Rainer et alii [2]. 
A figura 2 mostra os valores do preço do carvão baixo volátil e da penalidade por $0,10 \%$ de enxofre, para carvões que contenham entre $0,3 \%$ e $1,0 \%$ de enxofre, no período compreendido entre janeiro e julho de 2014.

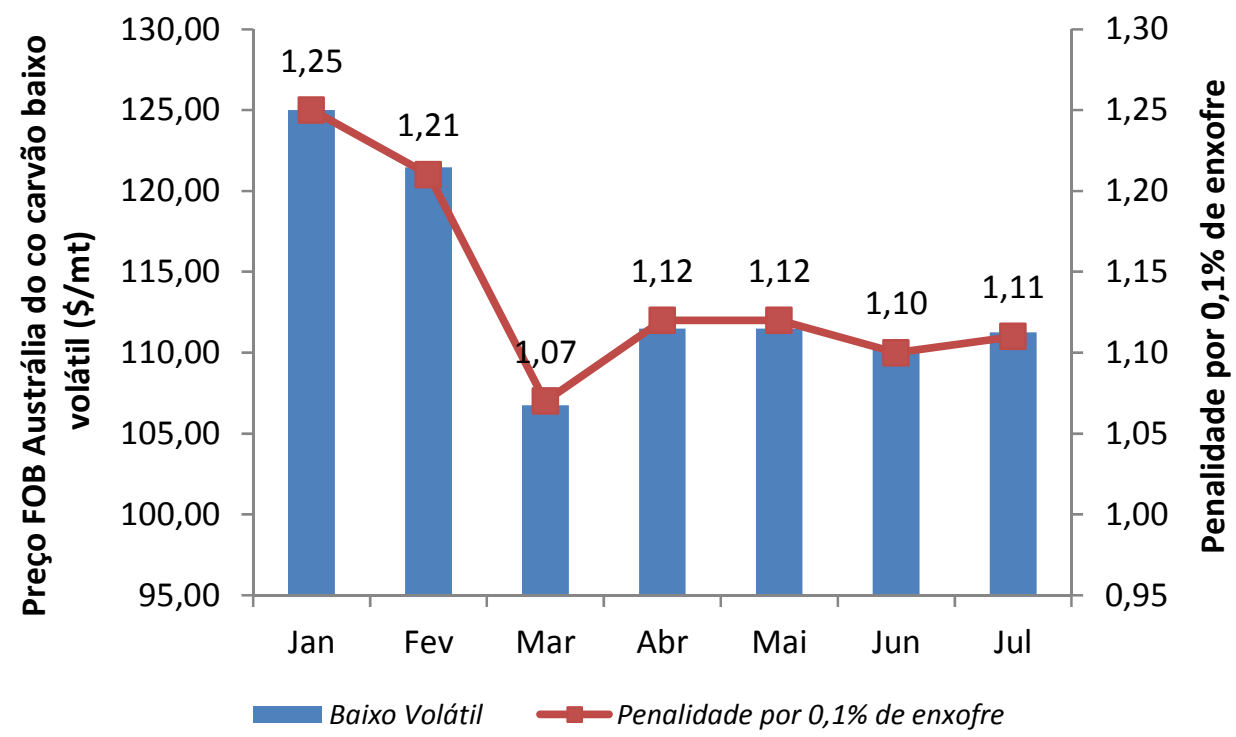

Figura 2. Preço do carvão baixo volátil e penalidade por $0,10 \%$ de enxofre.

Este estudo foi conduzido de forma a avaliar o impacto do teor de enxofre dos carvões metalúrgicos nos seus valores em uso, considerando utilização de escória sintética como agente dessulfurante e o impacto de variáveis operacionais e econômicas nos valores em uso. Os resultados obtidos são comparados com os valores de referência do mercado.

\subsection{Dessulfuração com Escória Sintética}

A capacidade de uma determinada escória absorver enxofre é avaliada por meio do coeficiente $\mathrm{Cs}$, ou capacidade de sulfeto. A equação 1 representa a dessulfuração com escórias sintéticas e a presença de enxofre sob a forma de sulfeto na escória. Nesta equação os íons entre parênteses representam a incorporação dos mesmos à escória.

$\frac{1}{2} S_{2(g)}+\left(O^{2-}\right)=\frac{1}{2} O_{2_{(g)}}+\left(S^{2-}\right)$

A constante de equilíbrio da reação 1 é dada por:

$K_{S}=\frac{h_{S^{2^{-}}} \cdot \sqrt{p_{O_{2}}}}{\left(a_{O_{2^{-}}}\right) \cdot \sqrt{p_{S_{2}}}}$

$\mathrm{Na}$ qual, $K_{S}=$ constante de equilíbrio da reação 1 (adimensional)

$h_{S^{2^{-}}}=$atividade henriana do enxofre na escória (adimensional);

$P_{\mathrm{O}_{2}}=$ pressão parcial de equilíbrio do oxigênio (atm); 
$a_{\mathrm{O}_{2^{-}}}=$atividade do oxigênio na escória (adimensional).

$P_{S_{2}}=$ pressão parcial de equilíbrio do enxofre (atm);

Aplicando hi=\%i.fi na expressão 2, tem-se que

$$
K_{S}=\frac{(\% S) \times f_{S^{2-}} \times \sqrt{p O_{2}}}{\left(a_{O_{2^{-}}}\right) \cdot \sqrt{p S_{2}}}
$$

Transpondo os termos $f_{S^{2-}}$ e $\left(a_{O_{2^{-}}}\right)$para o lado esquerdo da equação, pode-se definir a capacidade sulfeto (Cs) como:

$C_{S}=K s \cdot \frac{a_{\left(O_{2^{-}}\right)}}{f_{\left(S^{2-}\right)}}=\frac{(\% S) \cdot \sqrt{p_{O 2}}}{\sqrt{p_{S_{2}}}}$

Na qual, $f_{\left(S^{2-}\right)}=$ coeficiente de atividade do enxofre na escória (adimensional); $\% S=$ percentual de enxofre na escória.

A expressão 4 necessita que se tenham disponíveis as pressões parciais de oxigênio e enxofre. Para contornar esta dificuldade altera-se o estado padrão para solução a 1\%. Aplicando a lei de Hess para as equações $5 ; 6$ e 7, tem-se como resultado a equação 8:

$\frac{1}{2} S_{2(g)}+\left(O^{2-}\right)=\frac{1}{2} O_{2_{(g)}}+\left(S^{2-}\right)$

$\frac{1}{2} O_{2_{(g)}}=\underline{O}$

$\underline{S}=\frac{1}{2} S_{2_{(g)}}$

$\underline{\mathrm{S}}+\left(\mathrm{O}^{2-}\right)=\left(\mathrm{S}^{2-}\right)+\underline{\mathrm{O}}$

As constantes de equilíbrio das reações 6 e 7 são dadas respectivamente por:

$$
\begin{aligned}
& K_{O}=\frac{\% O \times f o}{\sqrt{p O_{2}}} \\
& K_{S}=\frac{\% S \times f_{S}}{\sqrt{p S_{2}}}
\end{aligned}
$$

Isolando as pressões de oxigênio e enxofre do lado esquerdo de cada equação, tem-se: 
$\sqrt{p O_{2}}=\frac{\% O}{K_{O}}$

$\sqrt{p S_{2}}=\frac{\% S \times f_{S}}{K s}$

Substituindo as equações 11 e 12 na equação 4, tem-se:

$$
C_{S}=K s . \frac{a_{\left(O_{2^{-}}\right)}}{f_{\left(S^{2-}\right)}}=\frac{(\% S) \cdot \sqrt{p_{O 2}}}{\sqrt{p_{S_{2}}}}=(\% S) \times \frac{\frac{\% O}{K_{O}} \times f o}{\underline{\% S} \times f_{S}}
$$

A relação entre os teores de enxofre na escória e no metal líquido (gusa ou aço) é denominada partição de enxofre (Ls), expressa matematicamente como:

$$
L_{S}=\frac{(\% S)}{\underline{\% S}}
$$

Utilizando este conceito na expressão 13 decorre que:

$$
L s=\frac{K_{O} \times f_{S}}{\underline{0 O} \times f o \times K_{S}} \times C_{S}
$$

A partição de enxofre (Ls) de uma dada escória pode ser estimada em função de sua composição química. De acordo com Sosinsky [4] a capacidade de sulfeto de uma dada escória é correlacionada com sua basicidade ótica, conforme equação 16:

$$
\log \left(C_{S}\right)=\frac{22690-54640 \times \Lambda_{t h}}{T}+43,6 \times \Lambda_{t h}-25,2
$$

Na qual, $\Lambda_{t h}=$ basicidade ótica teórica.

A basicidade ótica teórica pode ser estimada por meio de uma correlação semiempírica proposta por Duffy \& Ingram e citada por Susaki [1]:

$$
\Lambda_{t h}=\frac{1}{1,36 \times\left(X_{i}-0,26\right)}
$$

Na qual, $X_{i}=$ eletronegatividade de Pauling.

Considerando uma mistura de óxidos, a qual é o caso das escórias siderúrgicas, aplica-se a seguinte equação:

$$
\Lambda_{t h}=\sum \Lambda_{t h_{i}} \times x_{i}
$$

Na qual, $x_{i}=$ fração catiônica equivalente. 
A fração catiônica equivalente do óxido de cálcio, por exemplo, para uma escória composta por $\mathrm{CaO}, \mathrm{Al}_{2} \mathrm{O}_{3}, \mathrm{SiO}_{2}$ e $\mathrm{CaF}_{2}$ é definida pela equação 19.

$$
x_{\mathrm{CaO}}=\frac{\mathrm{NCaO}}{\mathrm{NCaO}+3 \mathrm{NAl}_{2} \mathrm{O}_{3}+2 \mathrm{NSiO}_{2}+\mathrm{NCaF}_{2}}
$$

$\mathrm{Na}$ qual $\mathrm{N}=$ fração molar do óxido na escória sintética. Para os outros componentes da escória o cálculo é realizado de maneira análoga. A tabela 1 apresenta os valores de basicidade ótica teórica de óxidos de acordo com Nakamura citado por Susaki [1].

Tabela 1. Basicidade ótica teórica de óxidos de acordo com Nakamura citado por Susaki [1]

\begin{tabular}{|c|c|c|c|c|c|c|c|c|c|}
\hline Óxido & $\mathbf{N a}_{2} \mathbf{O}$ & $\mathbf{C a O}$ & $\mathbf{M g O}$ & $\mathbf{M n O}$ & $\mathbf{F e O}$ & $\mathbf{A l}_{2} \mathbf{O}_{3}$ & $\mathbf{S i O}_{2}$ & $\mathbf{P}_{2} \mathbf{O}_{5}$ & $\mathbf{C a F}_{2}$ \\
\hline$\Lambda_{t h}$ & 1,11 & 1,00 & 0,92 & 0,95 & 0,94 & 0,66 & 0,47 & 0,38 & 0,55 \\
\hline
\end{tabular}

\section{MATERIAIS E MÉTODOS}

Para determinar a partição de enxofre de uma dada escória sintética a partir da equação 15 além da capacidade de sulfeto é necessário determinar: $K_{O}, K_{S}$ e $f_{S}$. Os valores de $K_{O}$ e $K_{S}$ são determinados a partir da relação 19:

$$
K=\exp \left(\frac{-\Delta G^{o}}{R T}\right)
$$

Na qual, $\Delta G^{0}=$ variação de energia livre padrão da reação (cal $\left./ \mathrm{mol}\right)$

$\mathrm{R}=$ constante universal dos gases $(\mathrm{cal} /(\mathrm{K} . \mathrm{mol}))$

$\mathrm{T}=$ temperatura $(\mathrm{K})$

Para as reações 6 e 7 , respectivamente tem-se que:

$$
\begin{aligned}
\Delta G^{0} & =-27691-1,11 \times T \\
\Delta G^{0} & =-32311+5,61 \times T
\end{aligned}
$$

O valor do coeficiente de atividade do enxofre $\left(f_{s}\right)$ é calculado considerando uma composição típica de gusa, por meio da equação 21 :

$$
\log f_{S}=e_{S}{ }^{C} \times \% C+e_{S}^{S i} \times \% S i+e_{S}{ }^{M n} \times \% M n+e_{S}{ }^{P} \times \% P
$$

Na qual, es ${ }^{j}=$ parâmetro de interação de primeira ordem a $1873 \mathrm{~K}$, dos componentes (j) que afetam o comportamento do enxofre no ferro e nos aços. Os parâmetros de interação de primeira ordem a $1873 \mathrm{~K}$, dos componentes (j) que afetam o comportamento do enxofre no ferro e nos aços de acordo com Carvalho [5], são mostrados na tabela 2: 
Tabela 2. Parâmetros de interação de primeira ordem a $1873 \mathrm{~K}$, dos componentes (j) que afetam o comportamento do enxofre no ferro e nos aços, segundo Carvalho [5]

\begin{tabular}{|c|c|c|c|c|}
\hline$e_{i}^{j}$ & $\mathbf{C}$ & $\mathbf{S i}$ & $\mathbf{M n}$ & $\mathbf{P}$ \\
\hline $\mathrm{S}$ & 0,11 & 0,063 & $-0,026$ & 0,029 \\
\hline
\end{tabular}

A partir de um balanço de massa expresso pela equação 23 é possível determinar então o consumo específico de escória dessulfurante para dessulfurar uma determinada quantidade de ferro gusa a partir de um teor inicial de enxofre até um teor objetivado de enxofre.

$m_{G L} \times \% S_{G L}=\left(m_{E S C} \times \% S_{E S C}\right)+\left(m_{G L-D E S} \times \% S_{G L-D E S}\right)$

Rearranjando os termos tem-se que:

$m_{E S C}=\left(\frac{m_{G L} \times \% S_{G L}}{\% S_{G L-D E S}}-m_{G L-D E S}\right) \times \frac{1}{L_{S}}$

Os valores em uso dos carvões serão estimados considerando as configurações mostradas na tabela 3 , com o objetivo de compreender como variáveis operacionais e de preço afetam o impacto do teor de enxofre dos carvões no seu valor em uso, considerando dessulfuração com escória sintética.

Tabela 3. Matriz de simulações numéricas - dessulfuração do metal por meio da escória.

\begin{tabular}{|c|c|c|}
\hline Variável & Referência & Valor avaliado \\
\hline $\begin{array}{c}\text { Preço da escória } \\
\text { sintética }(\$ / \mathrm{t})\end{array}$ & 50 & 100 \\
\hline $\begin{array}{c}\text { Rendimento } \\
\text { dessulfuração }(\%)\end{array}$ & 80 & 70 \\
\hline \%S objetivado & 0,0150 & 0,0075 \\
\hline $\begin{array}{c}\text { Temperatura do gusa } \\
\text { líquido }\left({ }^{\circ} \mathrm{C}\right)\end{array}$ & 1350 & 1300 \\
\hline
\end{tabular}

Por meio de balanços de massa e utilização de correlações da literatura das etapas de coqueria e alto-forno e dos processos de dessulfuração, serão estimados para cada processo o preço de gusa dessulfurado do caso referência e o preço de gusa dessulfurado dos casos avaliados. O valor em uso do carvão é então definido como sendo aquele preço de carvão no qual a diferença entre o preço de gusa dessulfurado do caso referência e o preço de gusa dessulfurado dos casos avaliados é zero. Matematicamente tem-se:

$$
V I U=P_{C R}+\frac{\Delta C G}{m_{C Q} \times m_{C A R}}
$$

Na qual, $V I U$ = valor em uso do carvão (\$/t de carvão);

$P_{C R}=$ preço do carvão de referência ( $\$ /$ t de carvão);

$\triangle C G=$ variação de custo de gusa, ou seja, (Custo de gusa referência - Custo de gusa avaliado), em \$/t de gusa;

$m_{C Q}=$ massa de coque carregada no alto-forno (t de coque/t de gusa); 
$m_{C A R}=$ massa de carvão carregada na coqueria (t de carvão/t de coque).

\section{RESULTADOS E DISCUSSÃO}

A tabela 4 mostra os resultados de fração molar $(\mathrm{N})$ e fração catiônica equivalente (X) para os óxidos que compõem uma escória sintética com a seguinte composição: $\% \mathrm{CaO}=65 ; \% \mathrm{Al}_{2} \mathrm{O}_{3}=20 ; \% \mathrm{SiO}_{2}=10 ; \% \mathrm{CaF}_{2}=5$. A partir destas informações e da equação 17 é possível determinar a basicidade ótica $\left(\Lambda_{\text {th }}\right)$ dessa escória sintética.

Tabela 4. Fração molar e fração catiônica equivalente de uma dada escória dessulfurante

\begin{tabular}{|c|c|c|c|c|c|c|}
\hline $\begin{array}{c}\text { Escória } \\
\text { Dessulfurante }\end{array}$ & $\%$ & $\begin{array}{c}\mathbf{M}- \\
\text { Massa } \\
\text { molar } \\
(\mathbf{g} / \mathbf{m o l})\end{array}$ & $\begin{array}{c}\mathbf{n} \\
\text { (número } \\
\text { de } \\
\text { mols) }\end{array}$ & $\begin{array}{c}\mathbf{N} \\
\text { (fração } \\
\text { molar) }\end{array}$ & $\begin{array}{c}\text { Xi (fração } \\
\text { catiônica } \\
\text { equivalente) }\end{array}$ & $\boldsymbol{\Lambda}_{\text {thi }}$ \\
\hline$\% \mathrm{CaO}$ & 65 & 56,08 & 11,59 & 73,10 & 0,54 & 1,00 \\
\hline$\% \mathrm{Al}_{2} \mathrm{O}_{3}$ & 20 & 101,96 & 1,96 & 12,37 & 0,27 & 0,66 \\
\hline$\% \mathrm{SiO}_{2}$ & 10 & 60,09 & 1,66 & 10,50 & 0,16 & 0,47 \\
\hline$\% \mathrm{CaF}_{2}$ & 5 & 78,07 & 0,64 & 4,04 & 0,03 & 0,55 \\
\hline Total & 100 & - & 15,86 & 100,00 & 1,00 & - \\
\hline
\end{tabular}

$\Lambda_{t h}=\sum \Lambda_{t h_{i}} \times x_{i}=(1,00 \times 0,54)+(0,66 \times 0,27)+(0,47 \times 0,16)+(0,55 \times 0,03)=0,814$

Substituindo este valor na equação 15 , considerando uma temperatura do gusa de $1350^{\circ} \mathrm{C}$, tem-se:

$\log \left(C_{S}\right)=-3,1325$

$C_{S}=7,37 \times 10^{-4}$

Considerando a temperatura do gusa de referência, ou seja, $\mathrm{T}=1350^{\circ} \mathrm{C}(1623 \mathrm{~K}) \mathrm{e}$ substituindo este valor nas equações 20 e 21 , tem-se que:

$K_{S}=1327$

$K_{O}=9274$

Substituindo os parâmetros de interação do enxofre (tabela 3) e a composição típica de gusa considerada ( $\% \mathrm{C}=4,50 ; \% \mathrm{Si}=0,40 \% ; \% \mathrm{Mn}=0,40 ; \% \mathrm{P}=0,080)$ na equação 22, tem-se que:

$\log f_{S}=0,11 \times 4,50+0,063 \times 0,40-0,026 \times 0,40+0,029 \times 0,080$

Assim, $f_{s}=3,252$. 
Substituindo os valores de $\mathrm{Cs}_{\mathrm{s}} K_{O}, K_{S}$ e $f_{S}$ na equação 15 , tem-se considerando banho com alto grau de desoxidação, ou seja, $\underline{\% \mathrm{O}}=0,0002$ e fo $=0,9$ :

$$
L s=\frac{K_{O} \times f_{s}}{\underline{0 O} \times f o \times K_{s}} \times C_{S}=\frac{9274 \times 3,252}{0,0002 \times 0,9 \times 1327} \times 7,37 \times 10^{-4}=93
$$

A tabela 5 exibe os resultados de penalidade estimada para cada $0,10 \%$ de enxofre adicional no carvão, com relação a $0,40 \%$ que é o percentual de enxofre de referência, considerando dessulfuração com escória sintética.

Tabela 5. Simulações realizadas na dessulfuração com escória sintética e penalidade estimada para $0,10 \%$ de $\mathrm{S}$ adicional

\begin{tabular}{|c|c|c|c|}
\hline Número & Simulação & $\begin{array}{c}\text { Condição de } \\
\text { referência }\end{array}$ & Valor em uso $\mathbf{( \$ / \mathbf { t } )}$ \\
\hline 1 & Caso base & - & 119,34 \\
\hline 2 & $\begin{array}{c}\text { Preço escória } \\
\text { sintética }=100 \$ / \mathrm{t}\end{array}$ & $50 \$ / \mathrm{t}$ & 118,90 \\
\hline 3 & Rendimento $=70 \%$ & $80 \%$ & 119,28 \\
\hline 4 & $\%$ S aço $=0,0075 \%$ & $0,0150 \%$ & 118,90 \\
\hline 5 & $\begin{array}{c}\text { Temperatura do gusa } \\
\text { líquido }=1300^{\circ} \mathrm{C}\end{array}$ & $1300^{\circ} \mathrm{C}$ & 118,55 \\
\hline
\end{tabular}

A figura 3 é a representação gráfica dos valores em uso dos carvões na dessulfuração com escória sintética. A linha preenchida representa o valor em uso de referência do mercado. Pode-se observar que considerando dessulfuração com escória sintética, existe uma variação pronunciada no valor em uso, com os valores ficando entre 118,55 e $119,34 \$$ /t, considerando o preço do carvão de referência igual a $120 \$ / t$. A condição que exerceu maior influência sobre o valor em uso do carvão foi a temperatura do gusa, pois a reação de dessulfuração é endotérmica $\left(\Delta \mathrm{H}^{\circ}>0\right)$, sendo portanto desfavorecida termodinamicamente considerando um gusa líquido com temperatura inferior ao caso referência. 


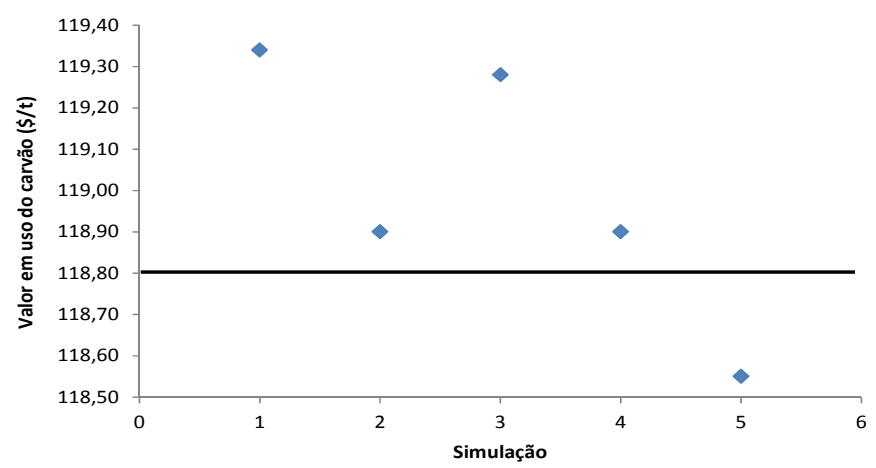

Figura 3. Valores em uso dos carvões na dessulfuração com escória sintética.

\section{CONCLUSÃO}

A dessulfuração de ferro-gusa e o impacto do teor de enxofre dos carvões no seu valor em uso foram avaliados considerando utilização de escórias sintéticas. Os resultados mostraram que para 4 das 5 condições avaliadas os valores em uso dos carvões foram maiores que a referência do mercado. Considerando o preço do carvão de referência igual a 120,00 \$/t o valor em uso referência do mercado assumindo $0,10 \%$ de enxofre adicional no carvão é de 118,80 \$t. Nas simulações de dessulfuração com escória sintética os valores em uso estimados estão entre $118,55 \$ /$ t e $119,34 \$$ t. . O maior valor em uso foi obtido considerando preço de escória sintética igual a $50 \$ / t$, rendimento de dessulfuração igual à $80 \%$, percentual de enxofre objetivado no aço igual a 0,0150\% e temperatura do gusa líquido igual à $1350^{\circ} \mathrm{C}$. O menor valor em uso foi obtido considerando uma temperatura do gusa líquido de $1300^{\circ} \mathrm{C}$.

\section{Agradecimentos}

À Vale, na pessoa dos gerentes Guilherme Defendi e Paulo Freitas Nogueira, pelas oportunidades e incentivo. Ao professor Luiz Fernando Andrade de Castro, pela orientação técnica e disponibilidade. Ao CNPq, CAPES-PROEX e FAPEMIG. Ao professor Katsujiro Susaki, pelas contribuições e discussões técnicas.

\section{REFERÊNCIAS}

1 Susaki, K. Pré-Tratamento de Gusa. Nova Lima: Vale, 2009. 47p. Relatório.

2 RAINER, $\mathrm{H}$. et alii Estratégias de dessulfuração na fabricação de aços com oxigênio. MPT Edição Brasileira, v.2, p.24-32, 2013.

3 Pielet, Howard; Tsvik, George. Value-in-use model from iron ore through direct-reduced iron and electric arc furnace. Disponível em https://www.ariel.ac.il/sites/conf/.../2_96106.doc. Acesso em: 30/12/2014.

4 Sosinsky D.J., Sommerville I.D. The composition and temperature dependence of the sulfide capacity of metallurgical slags. Metallurgical Transactions B, v. 17B, p. 331-337, 1986.

5 Carvalho, J, L, R. Dados Termodinâmicos para Metalurgistas. Belo Horizonte. UFMG, 1977. 